\title{
RFID outline and RFID applied in library
}

\author{
Zhang Ran
}

\section{Hubei University Of Education}

\begin{abstract}
In recent years,based on the rapid development of Radio Frequency Identification (RFID) ,the technique has been being used in more and more realms gradually.RFID is different from conventional bar code and is popular for a large number of advantages. This article mainly introduces the RFID applied situation within library management.
\end{abstract}

Keywords:RFID,Self-check in library,The accuracy of self-check.

\section{浅析RFID技术以及RFID图书馆的应用}

\section{张燃}

湖北第二师范学院, 武汉, 湖北, 中国

\begin{abstract}
中文摘要：近年来，随着RFID技术的快速发 展，越来越多的领域开始使用这项技术。不 同于传统的条形码, RFID因为具有更多的优 点而受到人们的青睐。本文主要论述了RFID 技术参与图书馆管理的应用状况。
\end{abstract}

\section{关键词: 射频识别、图书馆RFID自助借还、} 自助借还准确性、自助借还安全问题

\section{RFID原理及影响概述}

RFID (Radio Frequency Identification) 作为一种非接触式的无线 通信自动识别技术, 中文名为射频识别, 又 名电子标签 (E-Tag), 可以通过射频信号识 别特定目标并读写对象数据。RFID的识别工 作不同于条形码, 其作为条形码的无线版本, 识别过程无须人工操作。一套RFID系统由 RFID标签 (RFID Tag) 和RFID阅读器 (RFID Reader) 组成, 其基本工作原理为由RFID阅
读器发射特定频率的无线电波给RFID标签, 以此驱动RFID标签的电路将内部的身份密码 传递出, 再由RFID阅读器接受此身份密码, 再通过中间软件将其传送至计算机程序进行 处理。它的特殊之处在于具有防磁、防水、 耐高温、不怕脏污、使用寿命长、读取距离 远、安全性系数高、存储容量大等属性, 同 时可以一次性读取大量射频标签, 可以透过 容器读取标签。其最早的用处可追溯到第二 次世界大战时期用于区分友敌的侦查系统 [1], 如今RFID的应用在不同的领域发挥不同 的作用。例如在物流领域用于仓库管理、生 产线自动化、日用品销售; 在交通运输领域 用于集装箱与包裹管理、高速公路收费与停 车收费; 在农牧渔业用于羊群、鱼类、水果 等的管理以及宠物、野生动物跟踪等。 [2]RFID为生产生活带来了方便与快捷, 具有 广大的市场空间。 


\section{RFID应用区别举例}

通过调查可知, RFID应用最广的是在物 流零售业领域, 而其对RFID在图书馆的应用 影响深远。[3] 图书馆向来保护读者隐私, 将 RFID应用在图书馆管理上的可行性曾引起 公众的重视。相对于在零售业领域应用 RFID，RFID应用在图书馆管理上有相同也有 诸多不同之处。二者相同之处在于都需要大 量的标签对应货物或图书, 因此大量的标签 必将导致标签价格下降。然而, 它们的不同 之处首先在于使用年限: 商品属于一次消费, 零售业物件的标签寿命要求较短; 图书具有 相当长的流通年限, 长至几十年, 所以需要 更长寿命的标签。[3]其次, 因为同样的原因, 零售标签只需读写几次, 而图书标签需具备 经受成千上万次的读写能力, 这对标签制造 工艺的要求也是不同的。此外，二者的区别 还体现在物流领域和应用类型上。图书馆相 对封闭的RFID应用范围对比零售业开放式 市场化RFID使用。图书RFID标签大多只在一 定范围内的图书馆之间流通, 而零售业RFID 标签往往总在变化中的地点之间流通, 需要 具备更大的信息容量。

\section{3. 图书馆RFID应用的价值}

首先, 对大部分图书馆来说, 书籍资料 总是在不断增加, 图书馆工作人员的规模并 没有随之增长, 以至于收发、运输、分拣和 排架的工作量越来越多, 使得图书管理员的 工作量加重, 也不能有效地完成工作。因此, 利用RFID技术解放图书馆管理员, 使其从以 上大部分繁琐工作中解放出来，回归到正常 的图书馆管理工作是非常有必要的。其次, 谈到图书馆的价值体现, 服务环节一直被认 为是重要的衡量标准。作为大众文化信息传 播中心，图书馆系统管理技术的提升对提高 其工作效率和质量起到至关重要的作用, 而 通过RFID技术可以对此有所帮助。实现图书 馆自助借还是利用RFID技术可以实现的基 本需求: 用户可以根据自己的阅读偏好和特 点、研究重点、历史记录而灵活自主地完成 由查询、检索、借阅以及复印组成的一系列 借书动作。这种方式不仅节约了人工、提高 了图书馆效率，同时保护了用户的隐私，也 使他们方便、满足了自我。

\section{4. 图书馆RFID自助借还机}

图书馆RFID自助借还系统需要借助于 计算机运行的windows操作系统和终端管理 软件。电源为整个系统供电。用户通过使用 带触摸屏的显示器查看操作信息的显示以及 完成信息的输入。感应区帮助实现RFID标签 的读取操作。无线网卡通过无线路由器与图 书管理服务器通讯。

流程设计是RFID自助借还机重要的设 计环节组合。为了提供更好的用户体验, 拥 有一个成熟的流程设计对 RFID自助借还机 来说是必须的。目前RFID自助借还机主要包 括借书、还书和续借功能。下图（图1）为笔 者分析总结的当前RFID图书馆界一个基本 的RFID自助借还机用户操作系统的概念模 型。不同的图书馆RFID安全方案可以根据该 流程的总体步骤做出改动以满足不同的使用 要求。主要流程可以分成如下步骤: 第一步: 选择语言, 用户可根据自身情况选择操作语 言; 第二步：请用户选择借书还是还书；第 三步：读取读者卡，这是一项重要的身份识 别步骤; 第四步: 输入密码, 用户可以自行 设定个性密码; 第五步: 按键确认, 用户此 时可以确认之前输入的信息; 第六步: 将要 进行借或还的书一次性放入指定感应区域 （此为RFID自主借还流程最重要的步骤）, 只需一次就可完成所有图书的借还; 第七步: 操作是否正常? 系统会识别书本情况供用户 判断, 若不正常则重试, 重试三次仍失败机 器可自动将处理结果提交到服务台; 第八步: 确认借书。至此流程结束。这个流程的最末 其实可以添加打印数据收据的功能, 方便存 根和下次借还书。笔者认为, 整个流程的优 点在于注重了个性化和隐私, 提供了语言选 择以及密码保护; 而提交确认信息以及重试 三次失败提交给服务台的服务也提高了 RFID自助借还机的借还准确性。

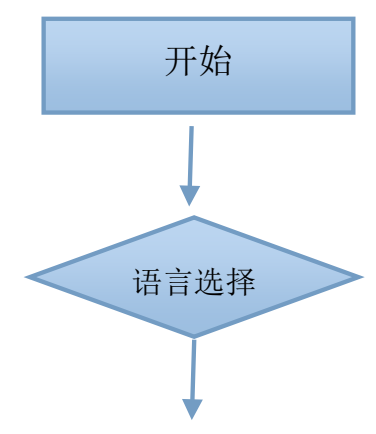




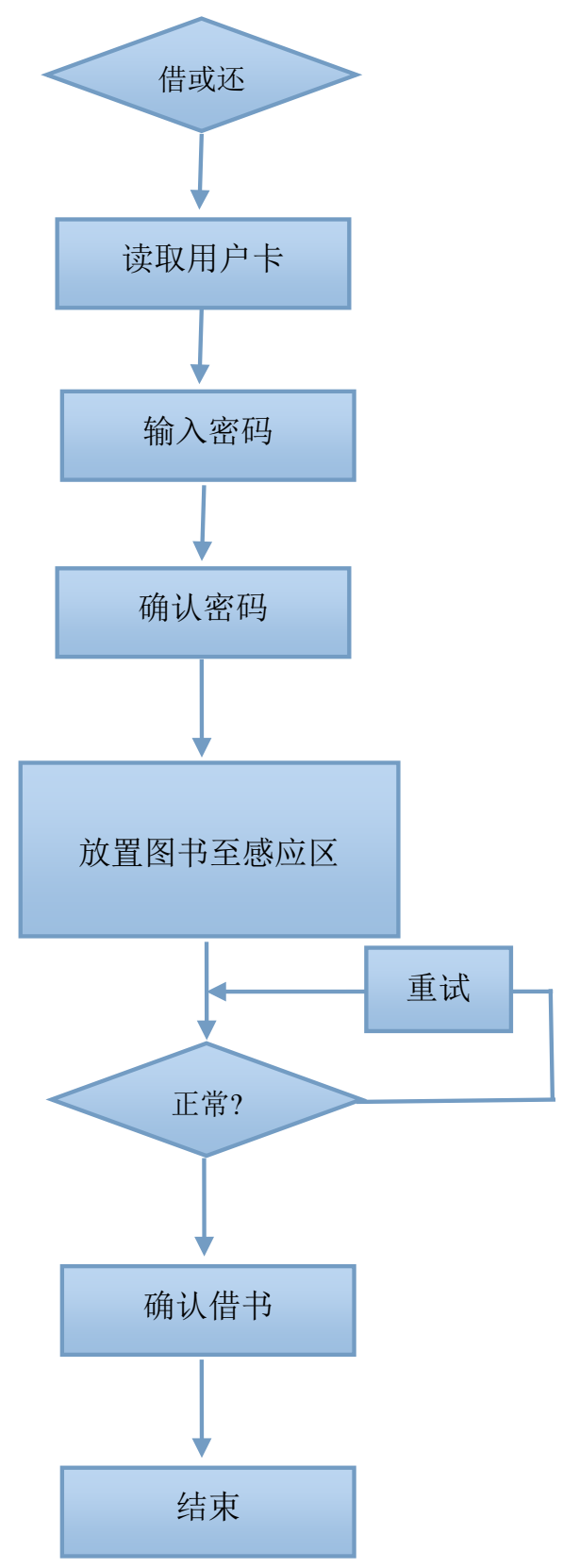

图1 RFID借书用户操作流程

\section{RFID自助借还机操作准确性的重要性}

不难发现, RFID自助借还机的使用受到 外在条件的限制, 比如RFID自助借还机器所 处空间位置等物理原因, 图书馆工作人员的 配合度; 内在因素可能存在人机交互是否简 单便利, 以及设备的处理速度和准确性等。 笔者认为提高RFID自助借还机的准确性是 非常重要的, 高准确率的RFID自助借还机能 让用户安心使用该机器, 也可吸引更多的用 户。推想而知, 如果过度追求RFID自助借还
机的借还速度而不考虑借还质量, 那么也许 会给自助借还的准确率打上折扣。

\section{6. 提高RFID自助借还准确性的措施}

通过调查、比较、分析，笔者总结了如 下四种可以提高RFID自助借还机准确性的 方法。

\section{1 限制一次借还书的数量}

作为一个非常有效的方法, 不仅存在于 运用了RFID技术的图书馆, 大部分图书馆都 有限制一次借还数量的规定。因为RFID标签 读取的特性，书本间的标签会因为互相干扰 而影响读取。一次放置的书本越多, 会减弱 机器一次全部读取的能力。因此限制读取书 本数量非常必要。

\section{2 设计良好的用户界面}

方便快捷的界面设计为用户带来优质的 UI即User Interface（用户界面）交互体验。 良好的界面设计能让用户在短时间内获取有 价值的信息，而不是复杂、无关且难懂的信 息, 因此力求自助机UI用词言简意赅。此外 可以采取适当的页面动态特效增强机器的体 验性, 为用户带来舒适的感官刺激, 集中用 户的注意力。

\section{3 屏幕即时显示读取结果并结合重试按钮}

当屏幕即时显示读取结果，应对错误的 读取结果可以通过重试按钮提高错误读取的 终止率。显示的结果应有借还书本的总数目 以及书名并力求简单醒目。这是一个给用户 判断读取正误的途径, 可以增加用户对自助 借还的信任程度。

\section{4 提供回执单}

通过数据回执单或E-mail提示向用户反 馈操作单，提供借还结果和时间，方便用户 查询借还记录、分析借还结果以及增加用户 对RFID自助借还机的信任度。

\section{RFID图书馆安全问题}

在2006年《加利福尼亚亚洲图书馆实践 RFID: 花费和收益》的调查中有这样一句话: RFID的安全是图书馆首选或次选目标。[3] 布庸置疑, 安全性好的图书馆才能长久地发 展下去, 因此安全问题可谓图书馆管理中的 
重中之重。传统的图书馆安全管理办法通常 是在每本馆藏书上贴上条形码作为其 “身份 证” , 并贴上磁条。到柜台办理借阅手续, 图书馆工作人员会对图书进行消磁。图书馆 的安全门在靠近未消磁的图书时会发出报警 音, 以确保每本书是以正常的借阅手续带出 图书馆。而涉及到RFID系统的图书馆安全管 理有更多的方案可供参考。笔者搜集了三个 可供参考的RFID系统图书馆安全方案, 下面 来分析比较这三个方案。

\subsection{RFID与磁条结合方案}

这种方案类似于传统的图书馆安全管理 方法, 还是以磁条, 而RFID负责流通和典藏。 这种方案的优点是, 磁条相对于RFID来说更 为隐蔽, 不轻易能被破坏。有实践证明出 RFID与磁条结合的安全方案如传统的磁条 方案一样, 也是有效的。但似乎存在一些问 题: 不能立马找出哪本书是非法借阅的; 磁 条间的混乱会造成一些错误报警; 与RFID相 结合的磁条安全方案需要支付额外的技术成 本。

\section{2 查询数据库方案}

此方案中安全门可以读取通过图书的标 示信息, 立即向图书数据库查询这本书的状 态是否是被借出, 并作出相应的反应。这种 方法的优点为只读型的RFID标签寿命长, 成 本较低。缺点为查询数据库的过程需要耗费 时间, 并需要强力的网络环境配合, 一旦中 间出了差错, 将大幅度影响工作效率。改进 方法是在数据库中建立已借出图书信息表, 并及时更新, 在图书通过安全门时, 能够强 有力地提高查询速度。

\subsection{EAS (Electronic Article Surveillance, 电 子商品防盗)安全位 (Security Bit) 方案}

此方案是把书本的状态在 RFID标签中 定位为 1 和 0 。 1 为安全位, 表示图书已被借出; 0 为危险位, 表示图书未被借出。在每次借还 操作时必须将危险位改为安全位, 因为安全 门会检索每次通过图书的状态位。这种方案 在安全门检索时速度比查询数据库方式要 快, 普遍被运用了RFID技术的图书馆接受。 不过由于标签经常要读写并用, 需要更高的 制作工艺, 所以成本较高。
上述三种方案，笔者更倾向于 EAS (Electronic Article Surveillance, 电子商品防 盗)安全位 (Security Bit) 方案。相比之下, 第一项RFID与磁条结合的方案虽在传统的 图书馆安全方案上做了改进, 但依旧低效率、 失误率高, 并没有达到运用RFID后理想的效 果。第二项查询数据库方案, 理念虽好, 但 涉及的环节太多, 对网络的依赖很大。而第 三种EAS安全位方案以便捷的方法解决了复 杂的问题, 体现了简单、直观的问题解决精 神。同时期待RFID技术的提升, 能降低RFID 标签制造成本, 使EAS安全卫方案得到普及。

\section{References}

[1]Connie K. H. , Lynne A.J. , Shai R. Radio Frequency Identification hand-book for librarians[M]. Libraries Unlimited,2007

[2]WU YX,Status and Prospects of RFID Technique,Microcomputer information,embedded system and SOC,2006,22:11-2

[3]CAI M. X. ,A Study on RFID Application in Libraries,National Library of China Publishing House,2010,2 